\title{
Enhanced acousto-optic interactions in a one-dimensional phoxonic cavity
}

\author{
I. E. Psarobas* and N. Papanikolaou \\ Institute of Microelectronics, NCSR “Demokritos," GR-153 10 Athens, Greece
}

N. Stefanou

Section of Solid State Physics, University of Athens, Panepistimioupolis, GR-157 84 Athens, Greece

B. Djafari-Rouhani

Institut d'Électronique, de Microélectronique et de Nanotechnologie, Université de Lille 1, 59655 Villeneuve d'Ascq, France

B. Bonello

Institut des NanoSciences de Paris, CNRS UMR 7588, Université Pierre et Marie Curie, 140 rue de Lourmel, 75015 Paris, France

V. Laude

Institut FEMTO-ST, Université de Franche Comté, CNRS, ENSMM, Besançon, France

(Received 5 September 2010; published 17 November 2010)

\begin{abstract}
We report on the occurrence of strong nonlinear acousto-optic interactions in a one-dimensional model phoxonic cavity that supports, simultaneously, photonic and phononic localized resonant modes, by means of rigorous electrodynamic and elastodynamic calculations. We show that these interactions can take place when photons and phonons of long lifetime are confined in the same region of space and lead to enhanced modulation of light by acoustic waves through multiphonon exchange mechanisms.
\end{abstract}

DOI: $10.1103 /$ PhysRevB.82.174303

PACS number(s): 42.70.Qs, 43.40.+s, 78.20.hb, 63.20.Pw

\section{INTRODUCTION}

Phoxonic crystals ${ }^{1-6}$ are appropriately designed structures with the ability to interface the transport properties of photons with phonons and vice versa. For example, such materials with dual spectral gaps, i.e., gaps for both optical and acoustic waves, can lead to applications which require better control of the acousto-optic (AO) interaction ${ }^{7,8}$ and, by all means, to impressive opportunities of manipulating photons with phonons. ${ }^{4}$ The AO effect has been widely used to process light signals in homogeneous materials for years. ${ }^{9}$ Moreover periodic multilayers, with or without defects, provide further possibilities for tailoring the AO interaction and allow one to observe distinct, interesting, and potentially useful physical phenomena. ${ }^{10}$ Thermal phonons in multilayer structures, can lead to strong Raman or Brillouin scattering, as a result of the simultaneous existence of localized phonon and photon modes in the same region of space. Potential coupling between such localized modes was anticipated in the case of a one-dimensional (1D) hypersonic phononic crystal $^{11}$ while strong Raman scattering was demonstrated in the case of a planar phonon cavity embedded within an optical nanocavity realized by a semiconductor heterostructure. ${ }^{12}$ In addition to the above, recent advances in cavity optomechanics ${ }^{13}$ added new means of exciting localized phononic modes in periodic zipperlike beams ${ }^{14}$ by the electromagnetic (EM) radiation pressure force. The anticipated dynamic backaction between mechanical motion and the cavity light field can further advance the reconfiguration and tunability of photonic systems. ${ }^{15}$ On the other hand, ultrafast acoustics techniques ${ }^{16,17}$ can probe in a straightforward and controllable manner the AO interaction in nanostructures ${ }^{18,19}$ by monitoring the transmission of light perturbed by a picosecond strain pulse. ${ }^{20}$ Such an approach has gained interest the last few years, either for identifying collective acoustic modes that could lead to light modulation in photonic crystals ${ }^{17,21}$ or, more importantly, to obtain ultrafast control of optical resonances in microcavity nanostructures. ${ }^{10,18}$

Inelastic light scattering (ILS) has proven to be of great value in the study of acoustic excitations and elastic behavior of various microstructures and nanoscaled systems via Brillouin and Raman experiments. The ILS spectra according to the AO effect accounts for the modulation of the electronic susceptibility of the medium caused by the acoustic modes. Its merit was clearly demonstrated in detailed studies, among others, on thin silicon membranes and silicon-on-insulator structures. $^{22}$ For samples consisting of a large number of layers, the problem is somewhat more complex, for one has to account for light scattering by a multilayer film with inhomogeneously modulated optical constants and thicknesses. Alternatively, time-domain methods have been developed, where a propagating acoustic wave with spatial variation changes the optical response of the structure. ${ }^{10,20}$ Obviously, the characteristic time scale of the strain field is much longer than the period of the light wave and, therefore, one can definitely treat the acoustic excitation as a quasistatic perturbation. In such a case the time evolution of the transmission of light, perturbed by the propagation of a strain field, clarifies the role of the AO effect on light modulation.

Slow-light effects on the AO interaction, and related applications, have been investigated both theoretically and experimentally. For example, it has been shown that ultraslow light can yield phase matching in optical fibers, which allows one to achieve strong coupling between high-quality acoustic waves of the fiber and multifrequency EM fields. ${ }^{23}$ Krishnamurthy and Santos have shown by means of numerical calculations that modulating a $1 \mathrm{D}$ optical cavity by a surface 


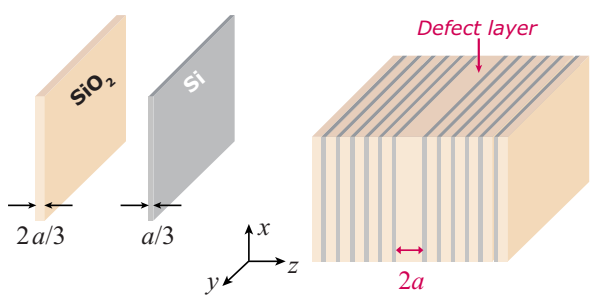

FIG. 1. (Color online) A 1D structure of homogeneous $\mathrm{SiO}_{2}$ and Si layers with a $\mathrm{SiO}_{2}$ defect layer in the middle forming a phoxonic cavity.

acoustic wave leads to enhanced AO diffraction. ${ }^{24}$ Moreover, it has been experimentally demonstrated that modulating a two-dimensional (2D) photonic crystal by a surface acoustic wave leads to enhanced $\mathrm{AO}$ diffraction thanks to slow-light propagation. ${ }^{25}$ The purpose of the present paper is to report a detailed theoretical investigation of AO effects in the simple case of a model 1D phoxonic cavity, realized by homogeneous $\mathrm{SiO}_{2}$ and $\mathrm{Si}$ layers, which is quite different from the above slow-light ideas. We discuss the differences in the interaction between resonant and nonresonant optical and acoustic modes, by analyzing the corresponding outgoing light waves both in time and frequency domains, and provide a consistent interpretation of the underlying physics.

\section{MODEL STRUCTURE AND METHOD OF CALCULATION}

We consider a 1D phoxonic crystal with a single defect, as shown in Fig. 1. A multilayer structure assembled by a periodic series of units described as $\left(\mathrm{SiO}_{2}-\mathrm{Si}\right)^{N}$ and a $\mathrm{SiO}_{2}$ layer of different width in the middle followed by the exact mirror series $\left(\mathrm{Si}-\mathrm{SiO}_{2}\right)^{N}$ of the same units stacked along the $z$ direction. We consider the number of units $N=6$ and each unit has a width $a$, where the widths of the $\mathrm{SiO}_{2}$ and $\mathrm{Si}$ layers are $2 a / 3$ and $a / 3$, respectively. The defect $\mathrm{SiO}_{2}$ layer has a width of $2 a$. The relevant material properties for $\mathrm{SiO}_{2}(\mathrm{Si})$ are characterized by the index of refraction $n=1.46(3.46)$, photoelastic coefficient $p_{12}=0.27(0.01)$, mass density $\rho$ $=2.20(2.33) \mathrm{g} / \mathrm{cm}^{3}$, and longitudinal sound velocity $c_{l}$ $=5970(8430) \mathrm{m} / \mathrm{s}$.

For the theoretical calculations involved in the present work we employ the layer-multiple-scattering (LMS) method, which is well documented for both elastodynamics ${ }^{26}$ and electrodynamics. ${ }^{27}$ This method constitutes a powerful tool for an accurate description of the acoustic and the optical response of composite structures comprised of a number of different layers having the same 2D periodicity in the $x-y$ plane (parallel to the layers). An advantage of the method is that it does not require periodicity in the $z$ direction (perpendicular to the layers). For each layer, the method calculates the appropriate transmission and reflection matrices $\mathbf{Q}^{\mathrm{I}}$ and $\mathbf{Q}^{\text {III }}$, respectively, for a plane wave of given frequency incident on the layer with given $x-y$ component of the wave vector, $\mathbf{k}_{\|}$, from $z \rightarrow-\infty$, i.e., with $k_{z}>0$, as well as the corresponding matrices $\mathbf{Q}^{\mathrm{IV}}$ and $\mathbf{Q}^{\mathrm{II}}$ for incidence from $z \rightarrow \infty$, i.e., with $k_{z}<0$. Explicit expressions for these $Q$ matrices can be found elsewhere. ${ }^{26,27}$ The transmission and reflection
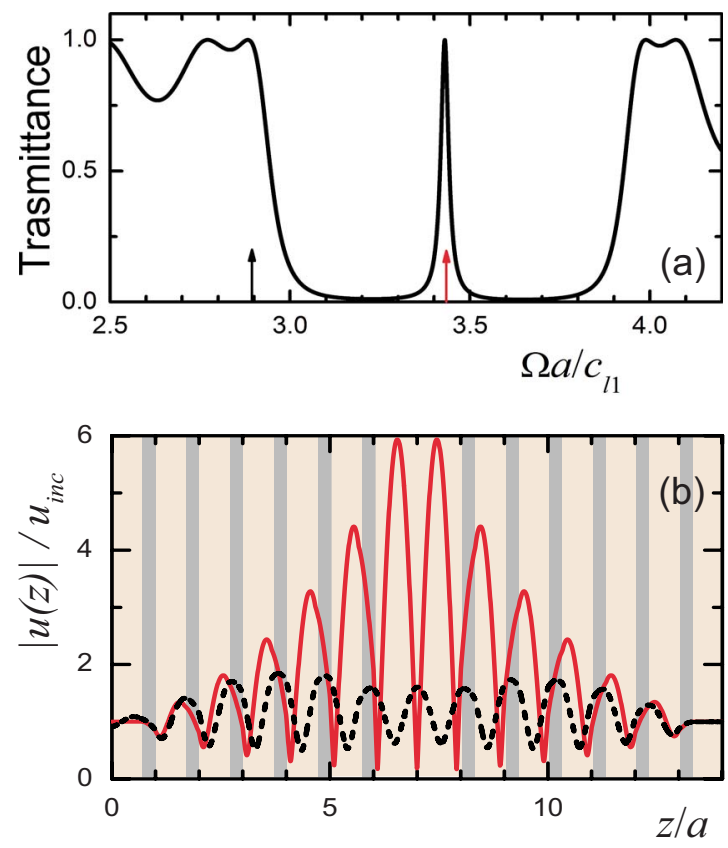

FIG. 2. (Color online) (a) Phononic transmission spectrum of a compressional acoustic wave normally incident on the phoxonic crystal of Fig. 1. (b) Displacement amplitude profile, normalized to the input displacement level $u_{i n c}$, at two selected frequencies marked by the arrows in Fig. 2(a): $\Omega_{1} a / c_{l 1}=3.43$ (at resonance, solid line) and $\Omega_{2} a / c_{l 1}=2.89$ (off-resonance, dashed line).

matrices of the composite structure are calculated from those of the constituent layers. We note that, in the case considered here, we deal with the simple situation where all layers are homogeneous.

The phononic response of the structure can be probed in practice by injecting an appropriate acoustic wave. In Fig. 2(a) we display the transmission spectrum for a compressional acoustic wave, at normal incidence, which was calculated by the full elastodynamic LMS method, ${ }^{26}$ assuming that the structure is embedded in a $\mathrm{SiO}_{2}$ matrix. We choose to represent the spectral range in units of dimensionless angular frequency $\Omega a / c_{l 1}$, where $c_{l 1}$ is the longitudinal sound velocity in $\mathrm{SiO}_{2}$, so that the results remain valid for any actual dimensions of the layers. Inside the frequency gap the defect mode manifests itself as an acoustic resonance of quality factor $Q=130$ at a frequency $\Omega_{1} a / c_{l 1}=3.43$. The $Q$ factor of course can be made arbitrarily high by including a large number of unit cells outside the localizing defect region, provided that dissipative losses are neglected. In Fig. 2(b) we depict the acoustic displacement amplitude profile at resonance, as well as at another frequency $\Omega_{2} a / c_{l 1}=2.89$ outside the gap, which will represent the off-resonance case. The compressional acoustic wave propagates along the $z$ direction and corresponds to an elastic displacement field $u(z, t)$ $=\mathfrak{R e}[u(z) \exp (-i \Omega t)]$ which induces a strain field $S(z, t)$ $=\partial_{z} u(z, t)$. These fields will actually determine the AO interaction with the EM wave. We note that we choose a compressional acoustic wave because, in this case, the AO effects are more pronounced.

The photonic response of the structure under consideration at normal incidence, in the absence of $\mathrm{AO}$ interaction, 

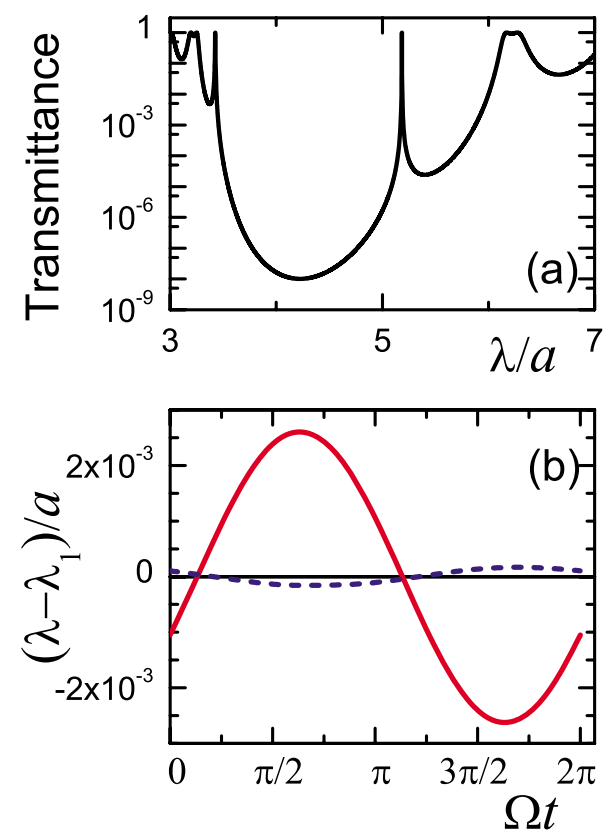

FIG. 3. (Color online) (a) Optical transmission spectrum of the phoxonic crystal of Fig. 1, at normal incidence, without the AO interaction. (b) Oscillatory behavior of the defect optical mode as a function of time for the acoustic excitation off-resonance (dashed line) and at resonance (solid line). The input displacement level is $u_{\text {inc }}=10^{-3} a / 3$ in both cases.

exhibits a frequency gap as well, with a high- $Q$ resonance $(Q=3100)$ inside the gap at a wavelength $\lambda_{1}=5.184 a$, as shown in the corresponding transmission spectrum displayed in Fig. 3(a), which was calculated by the full electrodynamic LMS method. ${ }^{27}$ For reasons explained above, the acoustic wave field will induce a quasistatic perturbation on the EM field. One can clearly identify two distinct contributions of the AO interaction. ${ }^{20}$ One is the interface (size) contribution due to the displacement of the boundaries of each layer, which translates to a variation in the thickness of the $n$th layer given by

$$
\Delta d_{n}(t)=u\left(z_{n}, t\right)-u\left(z_{n-1}, t\right),
$$

where $z_{n}, z_{n-1}$ define the unperturbed positions of the layer boundaries. The other contribution comes from the bulk AO effect, responsible for the inhomogeneous modulation of the dielectric function due to the spatial and temporal evolution of the strain field. According to the AO effect, the change in the index of refraction is given by ${ }^{9}$

$$
\Delta n(z, t)=-\frac{1}{2} n^{3}(z) p(z) S(z, t),
$$

where $p(z)$ is the appropriate photoelastic coefficient $\left(p_{12}\right)$ of the material at point $z$. The perturbation of the structural and optical parameters of the system, which will determine the AO interaction, are introduced by means of Eqs. (1) and (2). In general the continuous variation in the refractive index implied by Eq. (2) can be taken into account through a discretization approach by subdividing each layer into a large number of (homogeneous) elementary sublayers. However, in all cases examined here, about 100 sublayers in the whole structure, i.e., about seven sublayers per lattice constant, suffice to obtain excellent convergence.

\section{RESULTS AND DISCUSSION}

When the acoustic excitation is switched on, the optical transmission spectrum varies periodically in time with the period of the acoustic wave. To scale with reality we assume an input strain level of $10^{-3}$ at a nominal frequency $\Omega a / c_{l 1}$ $=3$, which is experimentally achievable. This corresponds to an incident acoustic signal with a displacement amplitude $u_{i n c}=10^{-3} a / 3$ and a strain level in the cavity that does not exceed $7 \times 10^{-3}$, which is below the material limit. Even though the overall spectral features and the position of the gap do not change much as time evolves, the position of the optical defect mode oscillates with a maximum relative wavelength shift, $\Delta \lambda / \lambda$, of $7 \times 10^{-5}$ when the acoustic excitation is off-resonance, in agreement with previous studies. ${ }^{10}$ However, if the acoustic excitation is at resonance, the amplitude of this oscillation increases by more than one order of magnitude and a larger shift $\Delta \lambda / \lambda$ of $1.4 \times 10^{-3}$ is obtained [see Fig. 3(b)]. It is worth noting that the dominant contribution in both cases comes from the interface effect [Eq. (1)]. The modulation of the optical defect mode by the acoustic excitation can be understood as follows. Assuming that the acoustic wave does not affect drastically the structure under consideration so that both photonic band gap and defect mode are maintained, the induced periodic variation in the refractive index and thickness of the defect layer results in a periodic oscillation of the position of the optical defect mode in the gap ${ }^{28}$ with the same period. If both optical and acoustic defect modes are involved, the corresponding photon-phonon interaction is enhanced because of the simultaneous concentration of the respective fields for a long time period in the defect region. In the wave picture this is manifested as a larger-amplitude oscillation of the position of the optical resonance. Correspondingly, in the particle picture, one expects strong inelastic light scattering with considerable probabilities for absorption and emission of many phonons by the photon, as will be explained below.

In general, the Lippmann-Schwinger equation for the electric field component of an EM wave scattered by the dielectric tensor perturbation, $\delta \bar{\varepsilon}(\mathbf{r}, t)$, induced by the $\mathrm{AO}$ interaction can be cast in the form of a Born series

$$
\begin{aligned}
\mathbf{E}(\mathbf{r}, t)= & \mathbf{E}^{0}(\mathbf{r}, t) \\
& +\frac{1}{c^{2}} \int d^{3} r^{\prime} \int d t^{\prime} \overline{\mathbf{G}^{0}}\left(\mathbf{r}, t ; \mathbf{r}^{\prime}, t^{\prime}\right) \delta \bar{\varepsilon}\left(\mathbf{r}^{\prime}, t^{\prime}\right) \partial_{t^{\prime}}^{2} \mathbf{E}^{0}\left(\mathbf{r}^{\prime}, t^{\prime}\right) \\
& +\frac{1}{c^{4}} \int d^{3} r^{\prime} \int d t^{\prime} \int d^{3} r^{\prime \prime} \int d t^{\prime \prime} \overline{\mathbf{G}^{0}}\left(\mathbf{r}, t ; \mathbf{r}^{\prime}, t^{\prime}\right) \delta \bar{\varepsilon}\left(\mathbf{r}^{\prime}, t^{\prime}\right) \\
& \times \partial_{t^{\prime}}^{2} \overline{\mathbf{G}^{0}}\left(\mathbf{r}^{\prime}, t^{\prime} ; \mathbf{r}^{\prime \prime}, t^{\prime \prime}\right) \delta \bar{\varepsilon}\left(\mathbf{r}^{\prime \prime}, t^{\prime \prime}\right) \partial_{t^{\prime \prime}}^{2} \mathbf{E}^{0}\left(\mathbf{r}^{\prime \prime}, t^{\prime \prime}\right)+\cdots,
\end{aligned}
$$

where $\mathbf{E}^{0}$ is the wave field in the absence of AO interaction, $\overline{\mathbf{G}^{0}}$ the corresponding Green's dyadic of the (unperturbed) structure while the time derivative of $\delta \bar{\varepsilon}$ has been neglected, since the variation in $\delta \bar{\varepsilon}$ is typically five orders of magnitude 

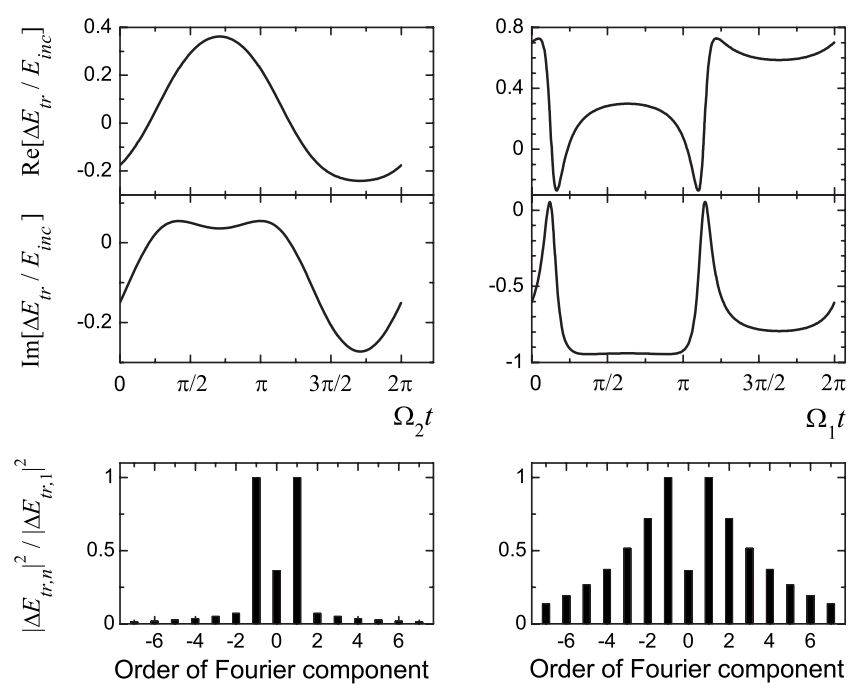

FIG. 4. Optical transmission through the phoxonic crystal of Fig. 1 under a continuous acoustic excitation of input level $u_{\text {inc }}$ $=10^{-3} a / 3$, for normally incident light at the resonance wavelength with electric field amplitude $E_{\text {inc }}$. Time variation in the perturbed transmitted optical field (upper diagrams) and intensities associated with the corresponding Fourier components (lower diagrams). The perturbation refers to the $\mathrm{AO}$ interaction with the acoustic excitation at the off-resonance frequency $\Omega_{2} a / c_{l 1}=2.89$ (left-hand panel) and at the resonance frequency $\Omega_{1} a / c_{l 1}=3.43$ (right-hand panel).

slower than that of the EM field. Assuming $\mathbf{E}^{0}$ to be a harmonic monochromatic wave of angular frequency $\omega, \delta \bar{\varepsilon}$ $=\Re \mathrm{Re}[\delta \bar{\varepsilon}(z) \exp (-i \Omega t)]$, and substituting into Eq. (3) the Fourier transform of the Green's dyadic, it can be shown after some straightforward algebra that Eq. (3) takes the form

$$
\mathbf{E}(\mathbf{r}, t)=e^{-i \omega t} \sum_{n=0, \pm 1, \cdots} \mathbf{E}_{n}(\mathbf{r}) e^{-i n \Omega t}
$$

Equation (4) tells us that the total outgoing wave (transmitted + reflected) consists, in general, of an infinite number of monochromatic beams with frequencies $\omega, \omega \pm \Omega$, $\omega \pm 2 \Omega, \ldots$, which are produced by elastic and inelastic photon scattering that involves absorption and/or emission of zero, one, two, ... phonons. Therefore, through a discrete Fourier analysis of the periodic evolution in time of the transmitted and reflected fields, one can obtain the intensities, $\left|\mathbf{E}_{t r, n}\right|^{2}$ or $\left|\mathbf{E}_{r f, n}\right|^{2}$, respectively, of the Fourier components, which determine the corresponding inelastic lightscattering cross sections.

We now assume an EM wave at the resonance wavelength, $\lambda_{1}$, incident normally on the phoxonic structure under consideration. The structure is continuously excited by a compressional acoustic wave, also propagating along the $z$ direction. In the left-hand panel of Fig. 4 we display the calculated change in the amplitude of the transmitted electric field, $\Delta E_{t r}$, induced by an acoustic wave with frequency $\Omega_{2} a / c_{l 1}=2.89$ (off-resonance). Both real and imaginary parts of $\Delta E_{t r}$ show a smooth sinusoidal-like variation with time. The corresponding Fourier spectrum is essentially dominated by the first-order term while all higher-order terms are at least one order of magnitude smaller. This is the behavior expected in a usual pump-probe or Brillouin/Ramanscattering experiment where single-phonon processes are involved and expansion up to first order in Eq. (3) is sufficient (Stokes and anti-Stokes components). ${ }^{20,29}$ The situation is the same at any wavelength off the optical resonance with the acoustic excitation being either off or at resonance. On the contrary, if the optical and acoustic modes involved in the inelastic light-scattering process are both simultaneously localized in the same region (defect region) for a long time period, strong interaction takes place. The scattering process is no longer linear on the AO coupling parameter and many terms are needed in the Born-series expansion of the Lippmann-Schwinger equation [Eq. (3)] which, in the particle picture, implies strong probability amplitudes for multiphonon absorption and emission processes. This is clearly demonstrated in the right-hand panel of Fig. 4 where both incident EM wave and acoustic excitation are at resonance. We observe sharp structures in $\Delta E_{t r}(t)$ when the oscillating optical resonance passes through the observation wavelength. This temporal variation is also reflected in the corresponding Fourier spectrum where, in addition to the strong first harmonics $(n= \pm 1)$, there are also significant higherorder terms that decrease slowly. Similar results are also obtained if we consider the reflected, instead of the transmitted, fields. It is also interesting to note in relation to the results presented in Fig. 4 that, though we considered the transmitted (or, equivalently, the reflected) fields induced only by the AO interaction, i.e., we subtracted the contribution of the unperturbed structure, there is still a small elastic-scattering component. This should be ascribed to multiphonon processes with simultaneous absorption and emission of the same number of phonons.

It is worth noting that our results apply to different regions of frequency, provided that the dimensions of the structural units are scaled accordingly. For example, choosing the lattice constant $a$ to be $300 \mathrm{~nm}$, the optical resonance is tuned at the telecom wavelength of $1.555 \mu \mathrm{m}$ while the acoustic resonance appears at a frequency $f=10.86 \mathrm{GHz}$. However, in order to compare with an actual experiment, wave damping, which arises from either material absorption or fabrication imperfections by transferring energy from the coherent beams to other degrees of freedom, should be taken into account. Though optical absorption in $\mathrm{SiO}_{2}$ and $\mathrm{Si}$ at infrared and longer wavelengths is generally small, attenuation at hypersonic frequencies may be rather strong and finite phonon lifetime effects can significantly downgrade the multiphonon exchange mechanism in the case of very-high- $Q$ acoustic resonances. In this respect, acoustic losses should be carefully considered in the design of functional phoxonic nanocavities. In the case considered here, our moderate- $Q$ acoustic cavity is sufficient to demonstrate enhanced AO interaction and multiphonon exchange mechanisms even if losses are included through typical complex propagation velocities $c_{l}(1-0.01 i)$.

\section{CONCLUSIONS}

In summary, we provided evidence for strong nonlinear $\mathrm{AO}$ effects in phoxonic cavities that support, simultaneously, 
acoustic and optical localized resonant modes, These effects, which can lead to efficient enhanced modulation of light with sound through multiphonon exchange mechanisms, have been demonstrated on a simple 1D model system of homogeneous $\mathrm{SiO}_{2}$ and $\mathrm{Si}$ layers. We calculated the time evolution of the scattered optical field when the structure is excited by an acoustic wave and analyzed the differences in the AO interaction between resonant and nonresonant modes providing a consistent interpretation of the underlying physics.

\section{ACKNOWLEDGMENT}

Financial support by FET-Open project TAILPHOX (Grant No. 233883) is acknowledged. *ipsarob@phys.uoa.gr

${ }^{1}$ T. Gorishnyy, M. Maldovan, C. Ullal, and E. L. Thomas, Phys. World 18(12), 24 (2005).

${ }^{2}$ M. Maldovan and E. L. Thomas, Appl. Phys. B: Lasers Opt. 83, 595 (2006).

${ }^{3}$ M. Maldovan and E. L. Thomas, Appl. Phys. Lett. 88, 251907 (2006).

${ }^{4}$ A. V. Akimov, Y. Tanaka, A. B. Pevtsov, S. F. Kaplan, V. G. Golubev, S. Tamura, D. R. Yakovlev, and M. Bayer, Phys. Rev. Lett. 101, 033902 (2008).

${ }^{5}$ S. Sadat-Saleh, S. Benchabane, F. I. Baida, M.-P. Bernal, and V. Laude, J. Appl. Phys. 106, 074912 (2009).

${ }^{6}$ N. Papanikolaou, I. E. Psarobas, and N. Stefanou, Appl. Phys. Lett. 96, 231917 (2010).

${ }^{7}$ M. M. de Lima, Jr. and P. V. Santos, Rep. Prog. Phys. 68, 1639 (2005).

${ }^{8}$ H. Ma, S. Qu, and Z. Xu, J. Appl. Phys. 103, 104904 (2008).

${ }^{9}$ A. Yariv and P. Yeh, Optical Waves in Crystals (Wiley, New York, 1984).

${ }^{10}$ X.-S. Qian, J.-P. Li, M.-H. Lu, Y.-Q. Lu, and Y.-F. Chen, J. Appl. Phys. 106, 043107 (2009).

${ }^{11}$ N. Gomopoulos, D. Maschke, C. Y. Koh, E. L. Thomas, W. Tremel, H.-J. Butt, and G. Fytas, Nano Lett. 10, 980 (2010).

${ }^{12}$ M. Trigo, A. Bruchhausen, A. Fainstein, B. Jusserand, and V. Thierry-Mieg, Phys. Rev. Lett. 89, 227402 (2002).

${ }^{13}$ M. Eichenfield, J. Chan, R. M. Camacho, K. J. Vahala, and O. Painter, Nature (London) 462, 78 (2009).

${ }^{14}$ J. Chan, M. Eichenfield, R. M. Camacho, and O. Painter, Opt. Express 17, 3802 (2009).

${ }^{15}$ M. Eichenfield, J. Chan, R. M. Camacho, K. J. Vahala, and O.
Painter, Nature (London) 459, 550 (2009).

${ }^{16}$ B. Bonello, B. Perrin, and C. Rossignol, J. Appl. Phys. 83, 3081 (1998).

${ }^{17}$ J.-F. Robillard, A. Devos, I. Roch-Jeune, and P. A. Mante, Phys. Rev. B 78, 064302 (2008).

${ }^{18}$ T. Berstermann, C. Brüggemann, M. Bombeck, A. V. Akimov, D. R. Yakovlev, C. Kruse, D. Hommel, and M. Bayer, Phys. Rev. B 81, 085316 (2010).

${ }^{19}$ P. M. Walker, J. S. Sharp, A. V. Akimov, and A. J. Kent, Appl. Phys. Lett. 97, 073106 (2010).

${ }^{20}$ O. Matsuda and O. B. Wright, J. Opt. Soc. Am. B 19, 3028 (2002).

${ }^{21}$ W. Y. Huang, W. Qian, and M. A. El-Sayed, Adv. Mater. 20, 733 (2008).

${ }^{22}$ J. Groenen, F. Poinsotte, A. Zwick, C. M. Sotomayor Torres, M. Prunnila, and J. Ahopelto, Phys. Rev. B 77, 045420 (2008).

${ }^{23}$ A. B. Matsko, Y. V. Rostovtsev, H. Z. Cummins, and M. O. Scully, Phys. Rev. Lett. 84, 5752 (2000).

${ }^{24}$ S. Krishnamurthy and P. V. Santos, Appl. Phys. Lett. 83, 2548 (2003).

${ }^{25}$ N. Courjal, S. Benchabane, J. Dahdah, G. Ulliac, Y. Gruson, and V. Laude, Appl. Phys. Lett. 96, 131103 (2010).

${ }^{26}$ R. Sainidou, N. Stefanou, I. E. Psarobas, and A. Modinos, Comput. Phys. Commun. 166, 197 (2005).

${ }^{27}$ N. Stefanou, V. Yannopapas, and A. Modinos, Comput. Phys. Commun. 132, 189 (2000).

${ }^{28}$ V. Karathanos, A. Modinos, and N. Stefanou, J. Phys.: Condens. Matter 6, 6257 (1994).

${ }^{29}$ J. He, B. Djafari-Rouhani, and J. Sapriel, Phys. Rev. B 37, 4086 (1988). 